Delft University of Technology

\title{
A 117DB in-Band CMRR 98.5DB SNR Capacitance-to-Digital Converter for Sub-NM Displacement Sensing with an Electrically Floating Target
}

Jiang, Hui; Amani, Samira; Vogel, Johan G.; Shalmany, Saleh Heidary; Nihtianov, Stoyan

DOI

10.1109/VLSIC.2018.8502363

Publication date

2018

Document Version

Accepted author manuscript

Published in

2018 Symposium on VLSI Circuits Digest of Technical Papers

\section{Citation (APA)}

Jiang, H., Amani, S., Vogel, J. G., Shalmany, S. H., \& Nihtianov, S. (2018). A 117DB in-Band CMRR 98.5DB SNR Capacitance-to-Digital Converter for Sub-NM Displacement Sensing with an Electrically Floating Target. In 2018 Symposium on VLSI Circuits Digest of Technical Papers (Vol. 2018-June, pp. 159160). [8502363] IEEE . https://doi.org/10.1109/VLSIC.2018.8502363

Important note

To cite this publication, please use the final published version (if applicable).

Please check the document version above.

\footnotetext{
Copyright

Other than for strictly personal use, it is not permitted to download, forward or distribute the text or part of it, without the consent of the author(s) and/or copyright holder(s), unless the work is under an open content license such as Creative Commons.

Takedown policy

Please contact us and provide details if you believe this document breaches copyrights.

We will remove access to the work immediately and investigate your claim.
} 


\title{
A 117dB In-band CMRR 98.5dB SNR Capacitance-to-Digital Converter for Sub-nm Displacement Sensing with an Electrically Floating Target
}

\author{
Hui Jiang, Student Member, IEEE, Samira Amani, Johan G. Vogel, Member, IEEE, Saleh Heidary \\ Shalmany, Member, IEEE, and Stoyan Nihtianov, Senior Member, IEEE
}

\begin{abstract}
This paper describes a high-performance Capacitance-toDigital Converter (CDC) for sub-nm displacement sensing with an electrically floating target. Intended to be integrated into a displacement sensor probe, the CDC consumes only $560 \mu \mathrm{W}$. It achieves $98.5 \mathrm{~dB}$ SNR in a $1 \mathrm{~ms}$ conversion time. With a sensing $\varnothing 8 \mathrm{~mm}$ probe and a $25 \mu \mathrm{m}$ stand-off distance from the target, it achieves $0.18 \mathrm{~nm}$ resolution. Moreover, it offers an in-band Common-mode Rejection Ratio (CMRR) higher than $117 \mathrm{~dB}$, providing decent electric field interference immunity.
\end{abstract}

Index Terms - Capacitance-to-Digital Converter (CDC); sub-nm displacement sensing; chopping; energy-efficient; electric field interference immunity; readout.

\section{INTRODUCTION}

Fully contactless displacement sensing with a sub-nm resolution is essential in high-precision mechatronic systems, such as imaging systems with target on a moving chuck [1]. Conventionally, the displacement sensor is positioned and aligned manually, which is time-consuming and costly. It becomes more difficult if the sensor is located at an inaccessible location in the machine. Thus, a standalone displacement sensor with autonomously position and align capability is of great interest. A displacement sensor, consisting of a thermal stepper system and an eddy-current based displacement-to-digital converter (DDC), has been proposed [2,3]. However, in order to mitigate the skin-effect and to attain sub-nm resolution within $1 \mathrm{~ms}$ of conversion time, a high excitation frequency $(>100 \mathrm{MHz})$ was applied [3]. This resulted in relatively high power dissipation $(>10 \mathrm{~mW})$ which can cause displacement errors due to self-heating, as the DDC has to be closely integrated with the eddy-current coil in the sensor head.



(a)

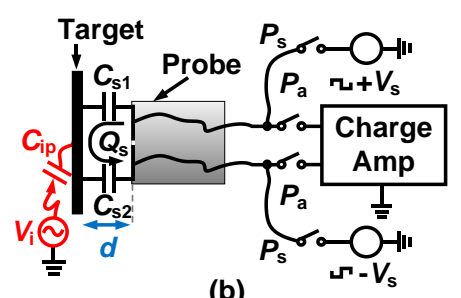

(b)
$[9,10]$ (Fig.1b). The sensor readout employs two identical capacitive sensing electrodes, positioned close to the target electrode. This results in two capacitors in series $\left(C_{\mathrm{s} 1}=C_{\mathrm{s} 2}=C_{\mathrm{s}}\right)$, whose magnitudes equally vary with the distance, $d$, to the target. By exciting the capacitors with anti-phase voltages $\pm V_{\mathrm{s}}$, the value of $C_{\mathrm{s}}$ can be obtained by measuring the charge $Q_{s}$. Compared to the conventional capacitive readout approach, it significantly reduces the area of the sensing loop and thus minimizes the error caused by the interfering magnetic fields [5]. However, such readouts with electrically floating targets are relatively power-hungry compared to conventional capacitive DDCs [7]. Moreover, any mismatch between the two sensing electrodes degrades the readout's CMRR and thus its electric field interference immunity [10]. To reduce the error caused by the misalignment of the probe and the target, the DDC needs a multi-channel input to obtain an out-of-plane tilt angle.

This paper presents a 3-channel capacitive DDC, with electrically floating target sensing capability [11], that overcomes these challenges while demonstrating an energy-efficiency comparable to the state-of-the-art capacitive DDCs which don't support the use of electrically floating targets [4-8]. With a sensing probe size smaller than that in [3], it achieves a $0.18 \mathrm{~nm}$ resolution in a $1 \mathrm{~ms}$ conversion time, at a $25 \mu \mathrm{m}$ stand-off distance to the target. Moreover, the interface consumes only $0.56 \mathrm{~mW}$, which is 16 times lower than the state-of-the-art eddy-current DDC [3].

\section{OPERATION PRINCIPLE}

\section{A. Thermal Slider Actuator}

To obtain an accurate displacement measurement, alignment of capacitive sensors is crucial. Self-alignment functionality integrated into the sensors reduces the cost of the conventional manual alignment, especially when they are installed at inaccessible locations in a machine. For this purpose, the Thermal Slider Actuator (TSA) was developed [1].

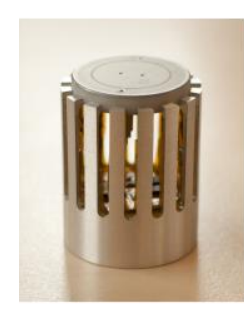

(a)

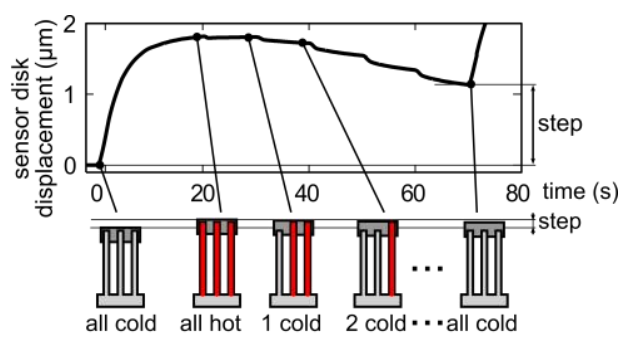

(b)
Fig.1a [4-8]. This means that the target electrode must be connected to the same ground as the interface electronics, which is a major drawback compared to the eddy-current DDCs, as this makes the capacitive sensor not fully contactless. Even worse, the ground loop incorporating the target and the input of the electronic interface is susceptible to electromagnetic interference and acts as a source of parasitic capacitance, both of which degrade the sensor's performance.

To minimize the loop while offering fully contactless displacement sensing, readouts with electrically floating targets have been proposed
Fig. 2. Thermal Slider Actuator (TSA) with twelve fingers (a) and a thermal cycle example and the resulting displacement profile (b) [1].

Fig. 2a shows the actuator structure of the TSA. The position adjustment of the sensing probe is based on the thermal actuation of a number of fingers that clamp the probe. Fig. $2 b$ shows an example of a thermal cycle of the fingers that leads to net displacement of the sensor probe. Heating of all elements together generates elongation by 
thermal expansion and moves the sensor probe to a new position. After that, the fingers are passively cooled one-by-one. When each finger cools, it slips over the probe surface, while the probe is kept in position by the other fingers. After the full thermal cycle, the probe has made a displacement step, while all fingers have cooled down and have returned to their original length. Because the actuation mechanism is fully switched off after positioning and the clamping is friction-based, the alignment obtained after actuation is maintained very stably [1].

\section{B. Measurement Method for Misalignment Correction}

Although the displacement with a floating target can be measured using two electrodes, misalignment of the probe and the target would lead to a sensing error. For this reason, a three-channel method is proposed. A third electrode is added to the probe (Fig. 3). Then, the probe capacitances can be measured through three channels $\mathrm{CH}$ : $\mathrm{CH} 1$ $\left(C_{\mathrm{s} 1}+C_{\mathrm{s} 2}\right), \mathrm{CH} 2\left(C_{\mathrm{s} 1}+C_{\mathrm{s} 3}\right)$, and $\mathrm{CH} 3\left(C_{\mathrm{s} 2}+C_{\mathrm{s} 3}\right)$. As the tilt error causes mismatching errors among the measured results of $\mathrm{CH} 1, \mathrm{CH} 2$, and $\mathrm{CH} 3$, the mismatching errors can then be used to minimize the tilt misalignment with the aid of self-alignment functionality of the TSA, matching the results of $\mathrm{CH} 1, \mathrm{CH} 2$, and $\mathrm{CH} 3$.

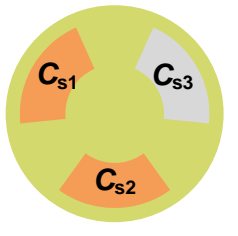

CH1

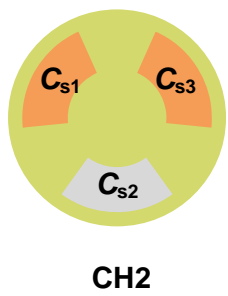

$\mathrm{CH} 2$

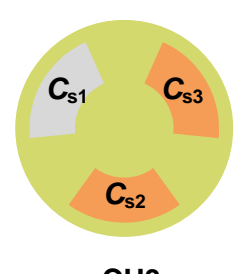

CH3
Fig. 3. Three electrode measurement for misalignment correction.

\section{Floating Capacitive Sensing Readout}

In practical implementations, the parasitic capacitance between the floating target and its surrounding environment ( $C_{\text {ip }}$ up to $500 \mathrm{fF}$ ) introduces a major challenge. It forms a path for interfering electric fields, primarily created by mains lines $(50 / 60 \mathrm{~Hz})$. As shown in Fig. $1 \mathrm{~b}$, the sensor front-end consists of a differential charge integrator with switched-capacitor excitation. $C_{\mathrm{s} 1}$ and $C_{\mathrm{s} 2}$ are periodically charged, delivering a signal charge $Q_{\mathrm{s}}=\left(\mathrm{C}_{\mathrm{s} 1} \cdot \mathrm{C}_{\mathrm{s} 2}\right) \cdot\left(\mathrm{C}_{\mathrm{s} 1}+\mathrm{C}_{\mathrm{s} 2}\right) V_{\mathrm{dd}}$ to the integrator. An interfering signal $V_{\mathrm{i}}$ then couples into the target electrode via $C_{\mathrm{ip}}$, resulting in a common-mode signal that will be rejected by the differential charge integrator.
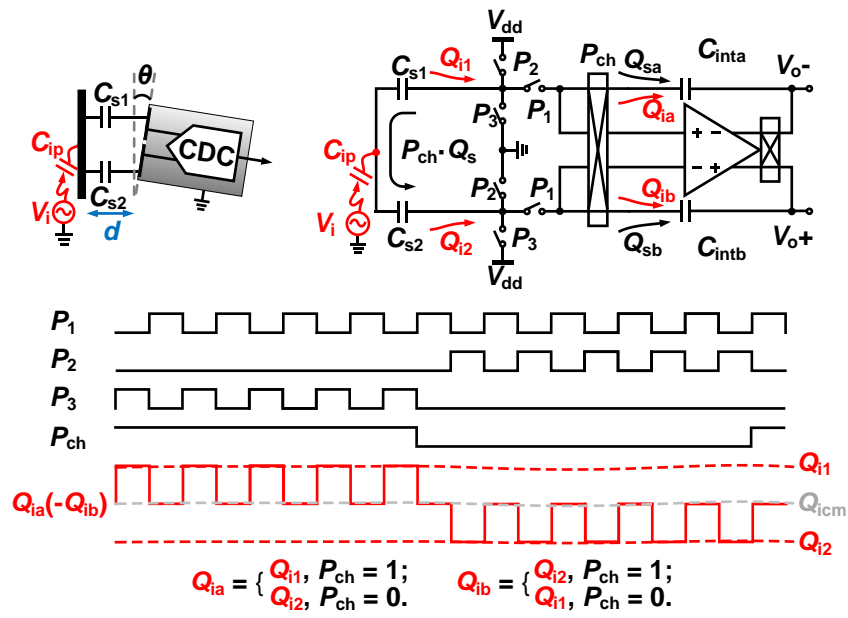

Fig. 4. Differential charge integrator with mismatched electrodes.

However, a mismatch between the sensing electrodes $\left(C_{\mathrm{s} 1} \neq C_{\mathrm{s} 2}\right)$, due to fabrication errors and/or alignment errors $(\theta)$, results in a deteriorated CMRR [10]. In this work, even with mismatched electrodes, a high CMRR is achieved by chopping the signal charge $Q_{\mathrm{i}}$ and the integrating capacitors $C_{\text {inta }}$ and $C_{\text {intb }}$ (see Fig. 4). The input switches $P_{1}-P_{3}$ are controlled by the chopping signal $P_{\mathrm{ch}}$, preserving the integration operation for $C_{\mathrm{s}}$ measurement. Due to the action of the input chopper, $C_{\text {inta }}$ and $C_{\text {intb }}$ continuously swap their position, such that $C_{\text {inta }}$ always accumulates negative charge ( $\left.Q_{\text {sa }}\right)$, while $C_{\text {intb }}$ always accumulates positive charge $\left(Q_{\mathrm{sb}}\right)$. At the same time, the mismatched interference charges $\left(Q_{\mathrm{i} 1}\right.$ and $\left.Q_{\mathrm{i} 2}\right)$, as well as the offset and the $1 / f$ noise of the integrator, are up-modulated to the out-of-band frequencies which will be filtered in digital domain.

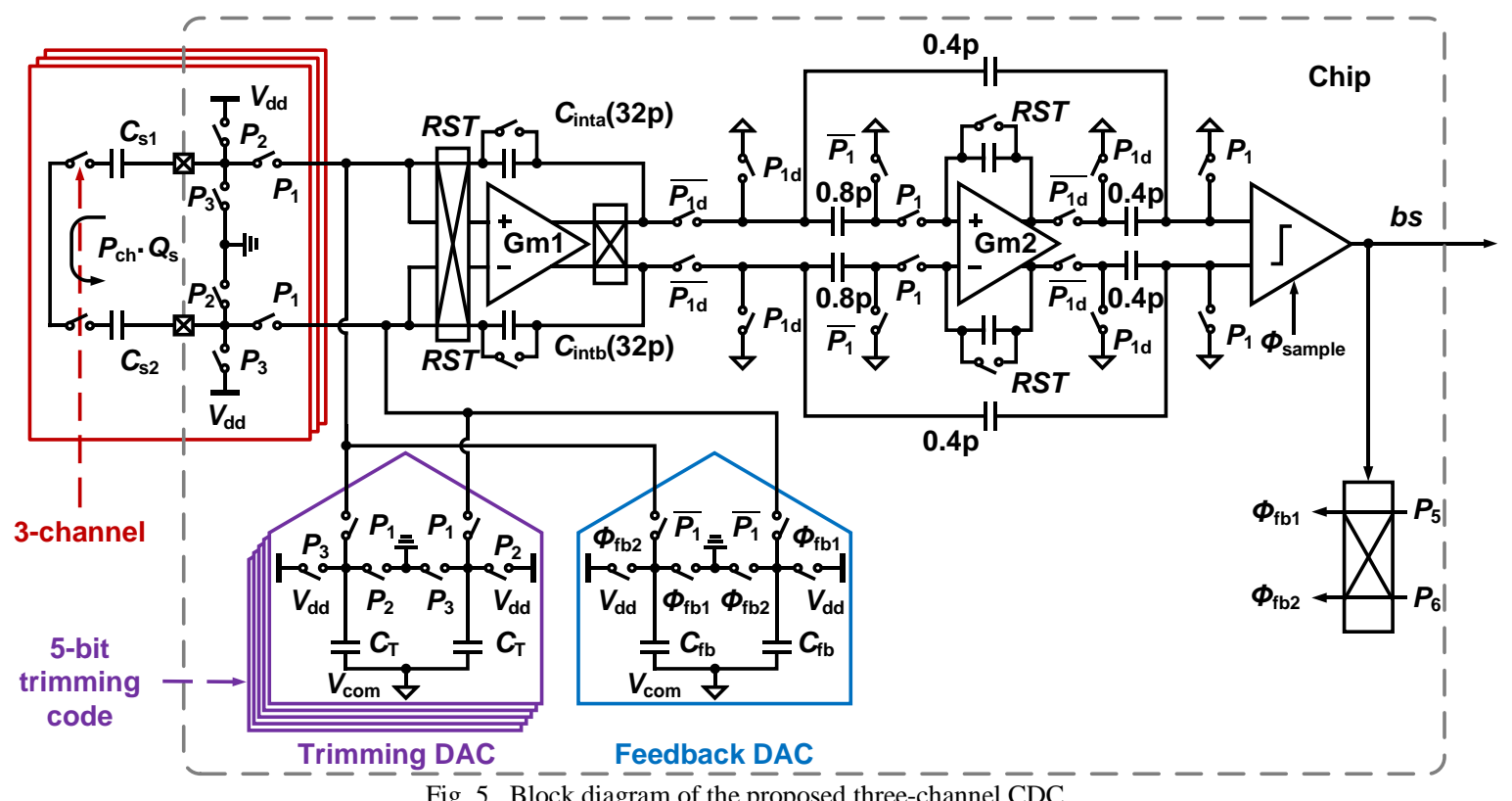

Fig. 5. Block diagram of the proposed three-channel $\overline{C D C}$ 
In contrast to [10], which first converts $Q_{\text {s }}$ into a proportional voltage and then digitizes it, this work directly embeds the charge integrator with sensing electrodes into a CDC, improving energy efficiency and reducing the number of potential error sources [5]. As shown in Fig. 5, the CDC is based on a second-order feedforward 1-bit sigma-delta modulator. The modulator's sampling frequency $f_{\mathrm{s}}$ is 1 $\mathrm{MHz}$, enough to achieve the target resolution with an OSR of 1000 , and the chopping frequency is set at $10 \mathrm{kHz}$ to minimize quantization noise fold-back [12]. The differential signal charge $Q_{\mathrm{s}}$ is counterbalanced by a feedback DAC, in which $C_{\mathrm{fb}}=10 \mathrm{pF}$. Depending on the output bitstream, it delivers a charge of $\pm C_{\mathrm{fb}} V_{\mathrm{dd}} / 2$. This charge-balancing results in an output bitstream with an average value of $C_{\mathrm{s}} / C_{\mathrm{fb}}$. To maximize the use of the CDC's available dynamic range, the modulator uses a programmable 5-bit trimming DAC to cancel the baseline capacitance, where $C_{\mathrm{T}-\max }=31 \mathrm{pF}$.

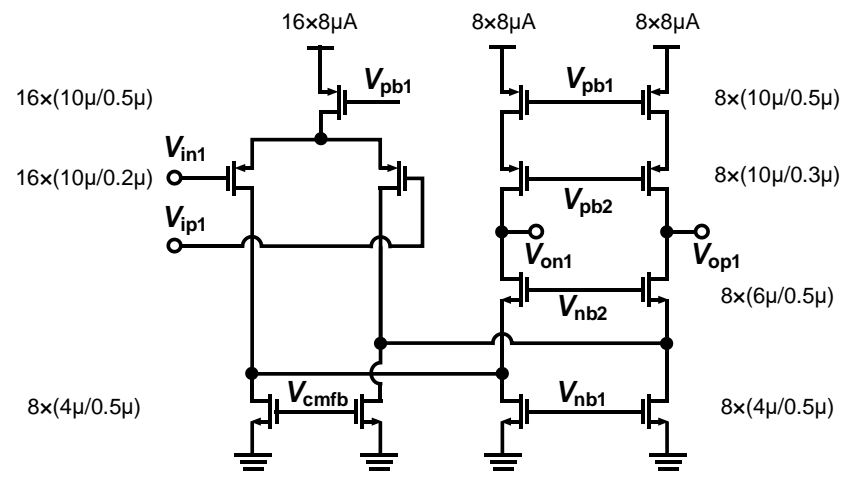

Fig. 6. The schematic of the 1st OTA and transistor-level parameters.

As shown in Fig. 6, the first integrator is based on a folded-cascode OTA with an $84 \mathrm{~dB}$ DC gain and a transconductance about $1 \mathrm{mS}$ to ensure the stability and accuracy for the worst case when the baseline cancelation capacitance is fully used. To achieve a lower intrinsic $1 / f$ noise corner frequency for lower chopping frequency, PMOS input pairs have been chosen. The biasing circuitry and common mode feedback circuitry are not shown for simplicity. A passive summation network at the input of the quantizer combines the outputs of second integrator with that of the first integrator via the feedforward capacitors as shown in Fig. 5. The quantizer is implemented as a dynamic latch proceeded by two preamplifiers. Each preamplifier provides a gain of 5 while consuming $3 \mu \mathrm{A}$.

\section{Measurement Results}


Fig. 7. Die micrograph of the CDC and power breakdown.

The CDC is realized in the $0.18 \mu \mathrm{m}$ standard CMOS process and tested with sensing electrodes designed on PCB. The chip occupies an active area of $0.5 \mathrm{~mm}^{2}$ and draws $313 \mu \mathrm{A}$, dominated by the $1 \mathrm{st}$ integrator, from a $1.8 \mathrm{~V}$ supply (Fig. 7). For flexibility, the decimation filter and digital controlling are implemented off-chip.

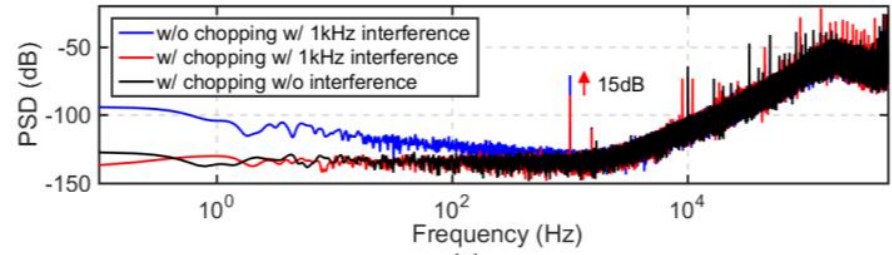

(a)



(b)

Fig. 8. PSD of the CDC's bitstream (a) and measured CMRR (b).

Fig. 8a shows the power spectral density (PSD) of the output bitstream. The CDC is thermal-noise-limited up to $1 \mathrm{kHz}$ and achieves $42 \mathrm{aF}$ resolution with a $10 \mathrm{pF}$ dynamic range in a $1 \mathrm{~ms}$ conversion time. The electric field interference rejection capability is tested by injecting interferences of different frequencies into the floating electrode through a $10 \mathrm{pF}$ capacitor $\left(C_{\text {ip }}\right.$ in Fig. 5$)$ driven by a sinusoidal voltage of $20 \mathrm{Vpp}$. This is equivalent to injecting a $400 \mathrm{Vpp}$ interference through a $500 \mathrm{fF}$ capacitor, an order of magnitude larger than what is expected in practical applications. The CMRR, defined as the ratio between the power of the input interference signal and its power at the CDC output, is used to evaluate the interference rejection performance. As shown in Fig. 8a, most of the interference power at 1 $\mathrm{kHz}$ is rejected by the differential architecture while chopping further suppresses the errors due to the mismatched electrodes $(<1 \%)$, reducing the interference tone by $15 \mathrm{~dB}$. As shown in Fig. $8 \mathrm{~b}$, the in-band CMRR with chopping is more than $117 \mathrm{~dB}$.
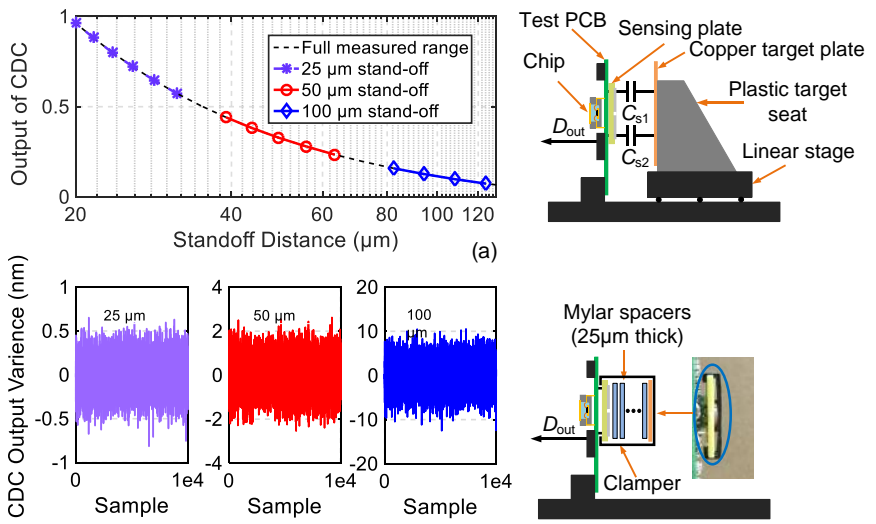

(b)

Fig. 9. Measured displacement transfer characteristics (a) and the outputs of the CDC (10,000 decimated samples) with different stand-offs along (b).

As shown in Fig. 9a, an electrically floating target is moved relative to the probe, back and forth, over a number of logarithmically spaced steps between $20 \mu \mathrm{m}$ and $130 \mu \mathrm{m}$ in two cycles and the displacement transfer characteristic is measured. To evaluate displacement resolution, the target electrode is firmly positioned using a clamp and Mylar spacer foil to avoid mechanical interference. The displacement 
sensing resolutions, obtained from the standard deviation of 10,000 decimated samples, are found to be $0.18 \mathrm{~nm}, 0.72 \mathrm{~nm}$, and $3 \mathrm{~nm}$ at standoffs of $25 \mu \mathrm{m}, 50 \mu \mathrm{m}$ and $100 \mu \mathrm{m}$, respectively (Fig. 9b). The real-time output of the CDC is shown in Fig. 10a. The measured backlash of the system is below $0.4 \%$. The relative inaccuracy is about $0.2 \%$ (Fig. 10b).

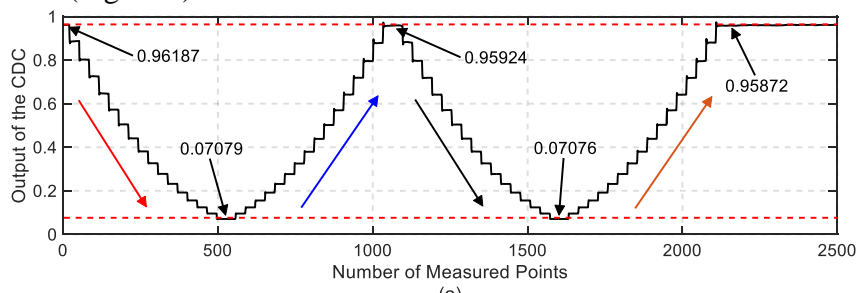

(a)

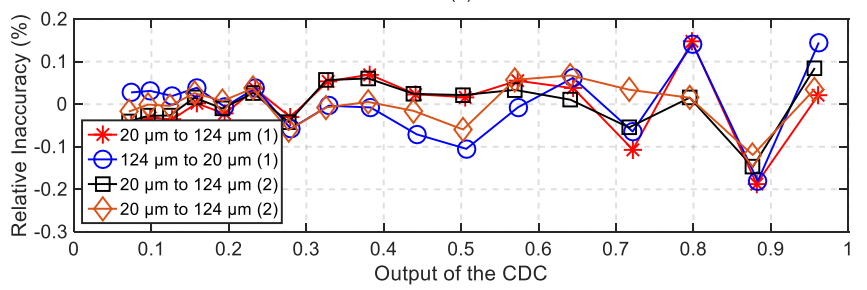

(b)

Fig. 10. Real-time measurement (a) and Relative inaccuracy (b).

The current consumption of the CDC versus supply voltage is shown in Fig. 11a. The CDC can work with a supply down to $1.5 \mathrm{~V}$, achieving a capacitance resolution better than $61 \mathrm{aFrms}$, which is mainly limited by the kT/C noise (Fig. 11b).

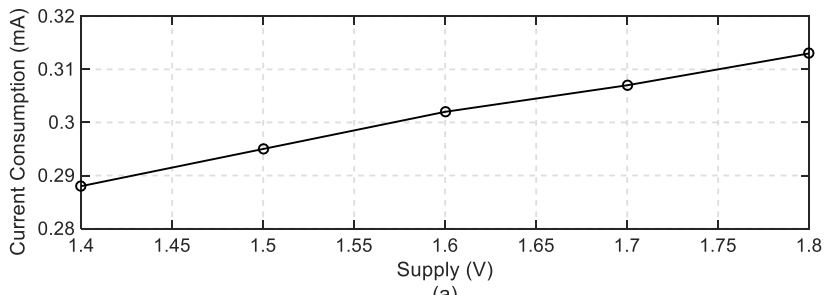

(a)



(b)

Fig. 11. Current Consumption vs. Supply (a) and Resolution vs. Supply (b).

TABLE I. Performance Summary and Comparison

\begin{tabular}{|c|c|c|c|c|c|c|}
\hline & This work & {$[5]$} & {$[6]$} & {$[7]$} & {$[8]$} & {$[10]$} \\
\hline Technology $(\mu \mathrm{m})$ & $\mathbf{0 . 1 8}$ & 0.35 & 0.35 & 0.16 & 0.16 & -- \\
\hline Floating target & Yes & No & No & No & No & Yes \\
\hline Power $(\mathrm{mW})$ & $\mathbf{0 . 5 6}$ & 14.9 & 0.76 & 0.003 & 0.014 & $121^{\star}$ \\
\hline Cap-range $(\mathrm{pF})$ & $\mathbf{0 - 1 0}$ & $\mathbf{8 - 1 2}$ & $6-22$ & $0-3.8$ & $0-8$ & $0-10$ \\
\hline SNR $(\mathrm{dB})$ & $\mathbf{9 8 . 5}$ & 84.8 & 102.6 & 114.6 & 80.9 & 81.3 \\
\hline Conversion time $(\mathrm{ms})$ & $\mathbf{1}$ & 0.02 & 10.5 & 100 & 6.86 & 0.1 \\
\hline FoM $(\mathrm{pJ} /$ Conv.-step) & $\mathbf{8 . 0}$ & 20.9 & 74 & 0.76 & 10.4 & $369^{\star}$ \\
\hline
\end{tabular}

${ }^{*}$ Without considering the power consumption of the ADC
Table I summarizes the performance of the CDC and the state-of-the-art. Compared to conventional CDCs [5-8], the proposed CDC offers floating target sensing capability, while achieving a similar energy-efficiency. Moreover, compared to a state-of-the-art inductive DDC [3], it achieves $10 \times$ better resolution with $16 \times$ less power consumption.

\section{CONCLUSION}

An energy-efficient CDC for a capacitive position sensor, with an electrically floating target, has been designed, implemented, and tested in a standard $0.18 \mu \mathrm{m}$ CMOS technology. The interface embeds the push-pull principle in a second-order $\Sigma \Delta$ Modulator. Experimental results show that it achieves a $98.5 \mathrm{~dB}$ SNR within $1 \mathrm{~ms}$ conversion time. The proposed CDC has an in-band CMRR higher than $117 \mathrm{~dB}$ and achieves comparable resolution FoM while being the only design offering floating target sensing capability.

\section{REFERENCES}

[1] O. S. van de Ven, J. G. Vogel, S. Xia, J. W. Spronck and S. Nihtianov, "Self-Aligning and Self-Calibrating Capacitive Sensor System for Displacement Measurement in Inaccessible Industrial Environments," in IEEE Transactions on Instrumentation and Measurement, vol. 67, no. 2, pp. 350-358, Feb. 2018.

[2] M. R. Nabavi, M. A. P. Pertijs and S. Nihtianov, "An Interface for Eddy-Current Displacement Sensors With 15-bit Resolution and $20 \mathrm{MHz}$ Excitation," in IEEE Journal of Solid-State Circuits, vol. 48, no. 11, pp. 2868-2881, Nov. 2013.

[3] V. Chaturvedi, J. G. Vogel, K. A. A. Makinwa and S. Nihtianov, "A 9.1 $\mathrm{mW}$ inductive displacement-to-digital converter with $1.85 \mathrm{~nm}$ resolution," 2017 Symposium on VLSI Circuits, Kyoto, 2017.

[4] A. Heidary and G. C. M. Meijer, "Features and Design Constraints for an Optimized SC Front-End Circuit for Capacitive Sensors With a Wide Dynamic Range," in IEEE Journal of Solid-State Circuits, vol. 43, no. 7, pp. 1609-1616, July 2008.

[5] S. Xia, K. Makinwa and S. Nihtianov, "A capacitance-to-digital converter for displacement sensing with $17 \mathrm{~b}$ resolution and $20 \mu$ s conversion time," 2012 IEEE International Solid-State Circuits Conference (ISSCC), San Francisco, CA, 2012.

[6] R. Yang, M. A. P. Pertijs and S. Nihtianov, "A Precision Capacitance-to-Digital Converter With 16.7-bit ENOB and 7.5-ppm $/{ }^{\circ} \mathrm{C}$ Thermal Drift," in IEEE Journal of Solid-State Circuits, vol. 52, no. 11, pp. 3018-3031, Nov. 2017.

[7] B. Yousefzadeh, W. Wu, B. Buter, K. Makinwa and M. Pertijs, "A compact sensor readout circuit with combined temperature, capacitance and voltage sensing functionality," 2017 Symposium on VLSI Circuits, Kyoto, 2017.

[8] Y. He, Z. Chang, L. Pakula, S. H. Shalmany and M. Pertijs, "A $0.05 \mathrm{~mm}^{2}$ $1 \mathrm{~V}$ capacitance-to-digital converter based on period modulation," 2015 IEEE International Solid-State Circuits Conference (ISSCC), San Francisco, CA, 2015.

[9] Lion Precision: Capacitive Sensor TechNote LT03-0022.

[10] X. Guo and S. N. Nihtianov, "A capacitive sensing technique for measuring displacement with one floating target electrode," 2010 IEEE International Conference on Industrial Technology, Mar, 2010, pp. $1565-1570$

[11] H. Jiang, S. Amani, J. G. Vogel, S. H. Shalmany and S. Nihtianov, "A 117DB in-Band CMRR 98.5DB SNR Capacitance-to-Digital Converter for Sub-NM Displacement Sensing with an Electrically Floating Target," 2018 IEEE Symposium on VLSI Circuits, Honolulu, HI, 2018.

[12] H. Jiang, C. Ligouras, S. Nihtianov and K. A. A. Makinwa, "A 4.5 $\mathrm{nV} / \sqrt{\mathrm{Hz}}$ Capacitively Coupled Continuous-Time Sigma-Delta Modulator with an Energy-Efficient Chopping Scheme," in IEEE Solid-State Circuits Letters, vol. 1, no. 1, pp. 18-21, Jan. 2018. 